\title{
NGHIÊN CÚU NHẬN THỨC BẢO VỆ THÔNG TIN RIÊNG TƯ CỦA NGƯỜI MUA HÀNG TRỤ̉C TUYẾN TẠI TP.HCM
}

\author{
BÙI THÀNH KHOA \\ Truờng Đại học Công nghiệp thành phố Hồ Chí Minh; \\ buithanhkhoai@iuh.edu.vn
}

Tóm tắt. Thương mại điện tử mang lại nhiều lợi ích cho người tiêu dùng nhưng cũng tiềm ẩn nhiều rủi ro như hàng hóa chất lượng kém, giao dịch gian lận và đặc biệt là những rủi ro về thông tin riêng tư của khách hàng. Bài báo nhằm phân tích sự tác động của các yếu tố (1) Quan tâm về riêng tư, (2) Niềm tin và (3) Nhận thức rủi ro đến nhận thức bảo vệ thông tin riêng tư khách hàng khi mua hàng trực tuyến. Nghiên cứu này được thực hiện với phương pháp hỗn hợp kết hợp phương pháp định tính và định lượng với mẫu khảo sát là 192 khách hàng mua hàng trực tuyến tại Thành phố Hồ Chí Minh. Kết quả nghiên cứu đã chỉ ra rằng nhận thức bảo vệ thông tin của người tiêu dùng vẫn còn thấp dù họ khác nhau về các yếu tố nhân khẩu học, cũng như tìm ra được các yếu tố niềm tin, cảm nhận rủi ro, quan tâm sự riêng tư có tác động đến nhận thức bảo vệ thông tin của khách hàng. Dựa trên kết quả nghiên cứu, tác giả cũng đưa ra một số gợi ý cho các doanh nghiệp kinh doanh trực tuyến để nâng cao kết quả hoạt động kinh doanh của mình.

Từ khóa. Nhận thức bảo vệ thông tin riêng tư, niềm tin, quan tâm sự riêng tư, rủi ro cảm nhận, thương mại điện tử/kinh doanh trực tuyến

\section{A STUDY ON THE ONLINE CUSTOMER PERCEPTION OF PRIVACY INFORMATION PROTECTION IN HO CHI MINH CITY}

\begin{abstract}
The electronic commerce brings not only many benefits for the consumers but potential risks as the bad quality goods, the fraudulent exchange, and especially, the dangers of the customers' privacy information. This paper aims to analyze the impact of (1) Privacy concern, (2) Trust and (3) Perceived Risk on Privacy Information Protection when the customer shop online. This research is carried out by the mixed method combined the qualitative and quantitative approach with the 192 respondents of the sample who buy online in Ho Chi Minh City. The result of research figured out that the consumer perception of privacy information protection is quite low although they are different in demographic factors; moreover, it found out that the trust, the perceived risk and the privacy concern of customer affect their perception. According to the research results, the author proposes some implications for the online businesses to improve their performance.
\end{abstract}

Keywords. Perception of Privacy information protection (PPIP), trust (TR), privacy concern (PC), perceived risk (PR), electronic commerce/online business.

\section{GIỚI THIỆ}

Sự ra đời và phát triển của mạng Internet toàn cầu mang đến nhiều sự tiện dụng trong hầu hết các lĩnh vực, trong đó mua sắm trực tuyến đã và đang trở thành xu hướng tiêu dùng mới của cuộc sống hiện đại. Hiện nay một bộ phận không nhỏ khách hàng lựa chọn và sử dụng mua sắm trực tuyến như là một công cụ hữu hiệu trong việc mua bán các hàng hóa, dịch vụ phục vụ cho đời sống của mình. Bên cạnh những lợi ích mà phương thức này mang lại, khách hàng đã và đang phải đối mặt với những trở ngại của việc mua sắm trực tuyến như khó kiểm định chất lượng hàng hóa, không tin tưởng người bán hàng, không có thẻ tín dụng hoặc các loại thẻ thanh toán qua mạng, cách thức mua hàng trực tuyến quá rắc rối.

Theo báo cáo của trang web Internetworldstats đến hết tháng 6/2016 tại Việt Nam, 51,5\% dân số đã sử dụng Internet trong cuộc sống hằng ngày, xếp thứ 15 trong 20 nước sử dụng Internet nhiều nhất trong thế giới [21]. Do cuộc sống của con người càng trở nên bận rộn, thời gian dành cho việc mua sắm truyền thống càng giảm bớt, khách hàng có xu hướng chuyển sang mua sắm trực tuyến vì sự tiện lợi và tiết kiệm thời gian. Bên cạnh sự xuất hiện ngày càng nhiều của các trang bán hàng trực tuyến thì các doanh nghiệp 
kinh doanh truyền thống cũng bắt đầu sử dụng các trang web để bán hàng song song với bán hàng truyền thống. Tính đến tháng 12/2015, số lượng các trang thương mại điện tử đăng ký với Cục trưởng Cục Thương mại điện tử và Công nghệ thông tin là 9.429 trang, tăng gần 50\% so với năm 2014 [4].

Để tồn tại trong nền kinh tế toàn cầu cạnh tranh cao, các doanh nghiệp phải tận dụng các công nghệ để thu thập, khai thác thông tin khách hàng, phân tích đặc điểm và hành vi của họ, xây dựng mối quan hệ với khách hàng hiện tại và rút ra những tiềm năng có thể phát triển từ khách hàng. Như vậy, thu thập thông tin về khách hàng là một công việc cần thiết cho các nhà quản lý để nắm bắt được nhu cầu, thị hiếu và sở thích của khách hàng. Nhưng điều đó cũng làm cho khách hàng rất do dự khi tham gia các giao dịch thương mại điện tử, nơi mà khách hàng được yêu cầu phải tiết lộ tin thông tin riêng tư như ngày tháng năm sinh, thu nhập, địa chỉ nhà, số điện thoại. Những mối quan tâm về thông tin riêng tư đang trở nên trầm trọng hơn bởi các lỗ hổng của Internet, ban đầu được thiết kế để dễ dàng truy cập và chia sẻ thông tin. Thông tin riêng tư có thể tác động tích cực cũng như cản trở sự phát triển của thương mại điện tử. Tạo nhận thức cho khách hàng rằng thông tin riêng tư của họ sẽ được bảo vệ cẩn thận và an toàn được xem là một yêu cầu quan trọng dẫn đến sự thành công của các doanh nghiệp kinh doanh trực tuyến. Đó cũng là một thách thức cho các nhà quản lý vì phải cân bằng giữa bảo đảm sự an tâm về sự riêng tư và thu thập thồng tin của khách hàng. Chính vì vậy việc NGHIẾN CÚUU NHẬN THỨC BẢO VỆ THÔNG TIN RIÊNG TƯ CỦA NGƯỜI MUA HÀNG TRỰC TUYẾN là phù hợp cho sự phát triển hiện nay của ngành thương mại điện tử Việt Nam và các dịch vụ trực tuyến.

\section{TỔNG QUAN CÁC TÀI LIÊUU NGHIÊN CÚU TRƯỚC ĐÂY}

Thương mại điện tử là việc hoàn thành bất kỳ một giao dịch nào bằng cách thông qua một mạng máy tính trung gian, bao gồm việc chuyển giao quyền sở hữu hay sử dụng hàng hóa, dịch vụ [30]. Thương mại điện tử bao hàm một loạt hoạt động kinh doanh trực tuyến cho các sản phẩm và dịch vụ, nó cũng là "bất cứ dạng nào của giao dịch kinh doanh trong đó các bên trao đổi qua lại điện tử hơn là sự trao đổi vật lý hay liên lạc trực tiếp vật lý" [26].

Thông tin riêng tư chính là tất cả các thông tin về cá nhân, bao gồm họ tên, ngày sinh và các mô tả khác, hoặc một số biểu tượng, mã, hình ảnh hoặc âm thanh được gán cho họ để nhận biết cá nhân (điều này cũng bao gồm thông tin không thể nhận biết được cá nhân, nhưng có thể dễ dàng nhận biết khi kết hợp với các thông tin khác). Ngoài ra, điều này cũng sẽ không giới hạn ở thông tin nhận dạng cá nhân, mà còn bao gồm tất cả các thông tin trình bày một dữ kiện, công nhận và đánh giá tính cách của cá nhân đó gồm hình dáng bề ngoài, tài sản, nghề nghiệp hoặc gia cảnh. Thông tin riêng tư là bất kỳ thông tin nào để xác định được hay có thể xác định được danh tính của một cá nhân cụ thể [7].

Bảo mật trực tuyến là một rào cản lớn đối với việc sử dụng có hiệu quả của Internet như một công cụ tiếp thị [5]. Jones và cộng sự cho rằng nhiều khách hàng không sẵn sàng để tham gia vào thương mại điện tử vì không chắc chắn về các vấn đề liên quan đến an ninh và sự riêng tư của dữ liệu giao dịch [15]. Các nhà nghiên cứu cũng lưu ý rằng nhận thức về những lỗi bảo mật hệ thống là nỗi sợ hãi lớn nhất cho người sử dụng trực tuyến khi dữ liệu có thể bị xem trộm bởi các bên thứ ba [22].

Bảo vệ thông tin là một chủ đề rộng bao gồm tất cả các vấn đề có liên quan đến lưu trữ và xử lý thông tin. Lĩnh vực nghiên cứu chính của bảo vệ thông tin gồm các vấn đề pháp lý như hệ thống chính sách, các quy định, yếu tố con người; các vấn đề thuộc tổ chức như kiểm toán xử lý dữ liệu điện tử, quản lý, nhận thức; các vấn đề kỹ thuật như: kỹ thuật mật mã, bảo mật mạng, công nghệ thẻ thông minh [3, 11 , 13]

Nhận thức là một phần quan trọng trong quá trình tư duy của con người và là một thành phần quan trọng để hiểu được hành vi của con người (Proctor and Proctor, 2007) [25]. Đó là cơ chế để một người đánh giá đầu vào từ bên ngoài, từ đó xác định phản ứng hành vi (Cooper, 2003) [6]. Vyskoc và Fibikova (2001) đã tiến hành một cuộc khảo sát về cách người dùng công nghệ thông tin nhận thức về bảo vệ thông tin [12]. Kết quả cho thấy người dùng không phải lúc nào cũng nghĩ giống như các chuyên gia bảo mật và họ cho rằng bảo mật thực sự chỉ có thể đạt được khi nhân viên của tổ chức hợp tác và hành xử một cách an toàn. Yenisey và cộng sự (2005) đã điều tra nhận thức của người dùng về an ninh trong thương mại điện tử và xây dựng hướng dẫn về nhận thức bảo vệ thông tin trong thương mại điện tử. Trong nghiên cứu này, sự nhận thức về bảo vệ thông tin được định nghĩa là cơ chế mà một người đánh giá và có biện pháp trước các mối đe dọa đối với an ninh thông tin, từ đó xác định phản ứng hành vi [33]. 
"Lý thuyết động cơ bảo vệ thông tin" của Rogers $(1975,1983)$ để giải thích hành vi nguy hiểm, rủi ro và để phát triển các giải pháp có hiệu quả để ngăn chặn hành vi nguy hiểm. Rogers (1975) đề xuất rằng động cơ của một người để bảo vệ mình khỏi các rủi ro phát sinh từ ba quá trình nhận thức: (1) nhận thức rủi ro có khả năng xảy ra cho mình, (2) nhận thức rủi ro sẽ nghiêm trọng, và (3) nhận thức hành vi bảo vệ có hiệu quả trong việc làm giảm nguy cơ được gọi là hiệu quả phản ứng. Để giải thích xu hướng tại sao cá nhân không tham gia vào các hành vi bảo vệ, Maddux và Rogers (1983) bồ sung cho lý thuyết động cơ bảo vệ thông tin bằng cách thêm ba yếu tố: (4) niềm tin của một cá nhân, (5) các chi phí phát sinh khi thực hiện một hành vi mong muốn, và (6) các lợi ích gắn liền với hành vi. Lý thuyết này nói rằng sáu thành phần là yếu tố quyết định quan trọng trong việc khơi gợi động lực bảo vệ thông tin của một cá nhân để kiểm soát tác hại hoặc nguy hiểm do những hành vi không an toàn.

Việc thiếu niềm tin trong bảo mật thông tin được xác định là một vấn đề lớn cản trở sự phát triển của thương mại điện tử trong nghiên cứu về mối quan tâm về bảo mật thông tin người dùng Internet [19]. Nghiên cứu tập trung vào ba vấn đề riêng biệt, nhưng liên quan chặt chẽ. Đầu tiên, dựa trên lý thuyết về các vấn đề xã hội, bài nghiên cứu đã cung cấp một khuôn khổ lý thuyết về mối quan tâm bảo vệ thông tin riêng tư của người dùng Internet (IUIPC). Thứ hai, bài nghiên cứu đưa ra các khái niệm đa chiều về IUIPC. Thứ ba là đề xuất và thử nghiệm một mô hình nhân quả về mối quan hệ giữa IUIPC và ý định hành vi hướng tới cung cấp thông tin riêng tư theo yêu cầu của một nhà tiếp thị. Nghiên cứu này đã tiến hành hai cuộc điều tra trên các lĩnh vực riêng biệt và thu thập dữ liệu từ 742 người trả lời từ các hộ gia đình. Các kết quả của nghiên cứu này chỉ ra rằng các yếu tố IUIPC trong đó bao gồm việc thu thập, kiểm soát, và các đặc tính tâm lý mong muốn nâng cao nhận thức về thông tin trong bối cảnh riêng tư trực tuyến. Nhận thức về tầm quan trọng của thông tin riêng tư của người tiêu dùng chắc chắn là một động lực thúc đẩy các phản ứng của họ để thực hiện một hành vi nào đó. Kết quả nghiên cứu này cho thẩy rằng niềm tin vào một nhà tiếp thị có thể giảm thiểu đáng kể rủi ro nhận thức và cuối cùng là sự miễn cưỡng của một khách hàng trong việc cung cấp thông tin riêng tư. Mối quan tâm riêng tư của khách hàng chắc chắn là một động lực thúc đẩy các phản ứng của khách hàng để họ thực hành một hành động nào đó.

Nghiên cứu "Xác định ảnh hưởng của quan tâm riêng tư trực tuyến đến hành vi bảo vệ thông tin riêng tư đối với trẻ vị thành niên" đã xác định yếu tố "mức độ quan tâm riêng tư trực tuyến" ảnh hưởng đến hành vi bảo vệ sự riêng tư [34]. Nghiên cứu cho thấy, sự đánh đổi giữa rủi ro và lợi ích sẽ dẫn đến hành vi bảo vệ sự riêng tư. Đặc biệt, nhận thức rủi ro cao do bị tiết lộ thông tin dẫn đến nhiều lo ngại về quyền riêng tư, trong khi nhận thức hơn về lợi ích được cung cấp trong trao đổi thông tin dẫn đến mối quan tâm ít hơn sự riêng tư. Mức độ quan tâm riêng tư dường như là một động lực quan trọng đối với các hành vi bảo vệ sự riêng tư chẳng hạn như tìm kiếm lời khuyên hoặc cố gắng không sử dụng một số trang web nhất định. Các hệ số hồi quy của các đánh giá nhận thức chỉ ra rằng nhận thức rủi ro là yếu tố quyết định quan trọng nhất trong việc giải thích các mức độ của mối quan tâm bảo mật sự riêng tư. Phát hiện này cung cấp cho các nhà giáo dục hiểu biết sâu sắc về lý thuyết sự riêng tư cho thanh thiếu niên.

Qua tóm tắt các nghiên cứu và căn cứ vào kết quả nghiên cứu định tính, người nghiên cứu quyết định sử dụng các biến niềm tin (Trust), nhận thức rủi ro (Perceived Risk) và quan tâm riêng tư (Privacy Concern) làm biến độc lập và biến nhận thức bảo vệ thông tin riêng tư (Perception of Privacy Information Protection) làm biến phụ thuộc cho nghiên cứu này.

\section{CÁC KHÁI NIÊMM NGHIÊN CỨU, GIẢ THUYÊTT VÀ MÔ HÌNH NGHIÊN CỨU}

Nhận thức về bảo mật trên Internet cung cấp cho người dùng đủ tự tin khi tham gia vào các giao dịch trực tuyến bằng cách giảm thiểu những lo ngại của họ về mối quan tâm riêng tư của họ. Với mức độ gia tăng kinh nghiệm sử dụng Internet, người dùng có nhiều khả năng nhận thức được các biện pháp bảo vệ thông tin riêng tư và có xu hướng quan hệ tích cực với ý định giao dịch thông qua trực tuyển [13]. Các công cụ được nhận thức để nâng cao khả năng bảo vệ sự riêng tư như phần mềm chống phần mềm gián điệp là yếu tố quyết định quan trọng nhất trong hành vi người dùng [16]. Theo nghiên cứu của Heng Xu và cộng sụ (2011) về mối quan tâm thông tin riêng tu và liên kết nhận thức cá nhân với chế độ đảm bảo bảo mật cho thấy rằng mối quan tâm riêng tư của khách hàng về việc có thể mất sự riêng tư như là kết quả của việc nhận thức về vấn đề phải tiết lộ thông tin cho một đại lý bên ngoài [32]. Culnan và Armstrong (1999) cho rằng mối quan tâm riêng tư là những quan tâm về những người có quyền truy cập vào thông tin khi sử dụng Internet. Các tác giả cũng chỉ ra rằng người tiêu dùng sẽ sẵn sàng hơn để tiết lộ 
thông tin và giao dịch nếu họ biết ai sẽ có quyền truy cập vào và sẽ được sử dụng thông tin của họ như thế nào. Tiết lộ thông tin riêng tư và giao dịch qua Internet có thể làm tăng mối quan tâm riêng tư vì công nghệ mang đến sự bất ổn lớn hơn về những người có quyền truy cập vào và sử dụng thông tin của người khác [8].

Giả thuyết H1: Mối quan tâm riêng tu tác động đồng biến đến nhận thức bảo vệ thông tin riêng tu trong giao dịch thuoong mai điện tư.

Trong thương mại điện tử, niềm tin được thành lập bởi người sử dụng dựa trên thông tin có sã̃n của công ty. Tin tưởng vào một trang web có thể tạo ra một thái độ tích cực đối với ý định cung cấp thông tin và giao dịch của khách hàng [17]. Niềm tin sẽ tác động tích cực đến ý định cung cấp thông tin và giao dịch của khách hàng [13]. Như vậy, một khách hàng tin tưởng trang web của doanh nghiệp sẽ sẵn sàng cung cấp những thông tin mà doanh nghiệp cần mà không có nhiều mối quan tâm về việc bảo vệ các thông tin đó. Một nghiên cứu với đối tượng sinh viên đại học cho thấy rằng niềm tin thấp đối với phương tiện truyền thông xã hội và các bên khác sẽ thúc đẩy việc sử dụng các cài đặt bảo mật để kiểm soát truy cập thông tin trên Facebook [10].

Giả thuyết H2: Niềm tin tác động nghịch biến đến nhận thức bảo vệ thông tin riêng tu trong giao dịch thuoong mại điện tư.

Rủi ro cảm nhận là sự không chắc chắn về những hậu quả tiêu cực khi sử dụng một sản phẩm hoặc dịch vụ. Rủi ro cảm nhận chính thức được định nghĩa là kết hợp của sự không chắc chắn và mức độ nghiêm trọng của các hệ quả liên quan $[1,2]$. Rủi ro cảm nhận ảnh hưởng tiêu cực vào ý định giao dịch thương mại điện tử [27]. Khi khách hàng cảm nhận được sự tăng lên của những rủi ro có thể xảy ra đối với họ như mất thông tin thẻ tín dụng, thông tin cá nhân, họ sẽ tăng cường bảo vệ thông tin riêng tư khi thực hiện các giao dịch. Rủi ro về cảm nhận sẽ tác động ngược chiều đến ý định cung cấp thông tin của khách hàng [19]. Một nghiên cứu tiếp thị về quyền riêng tư của người tiêu dùng cho thấy sự gia tăng trong nhận thức rủi ro của người sử dụng mà sẽ nâng cao nhận thức của các biện pháp bảo vệ sự riêng tư [24].

Giả thuyết H3: Rủi ro cảm nhận tác động đồng biến đến nhận thức bảo vệ thông tin riêng tu trong giao dịch thuơng mai điện tủ.

Từ những lý luận trên, tác giả đưa ra mô hình nghiên cứu như sau:

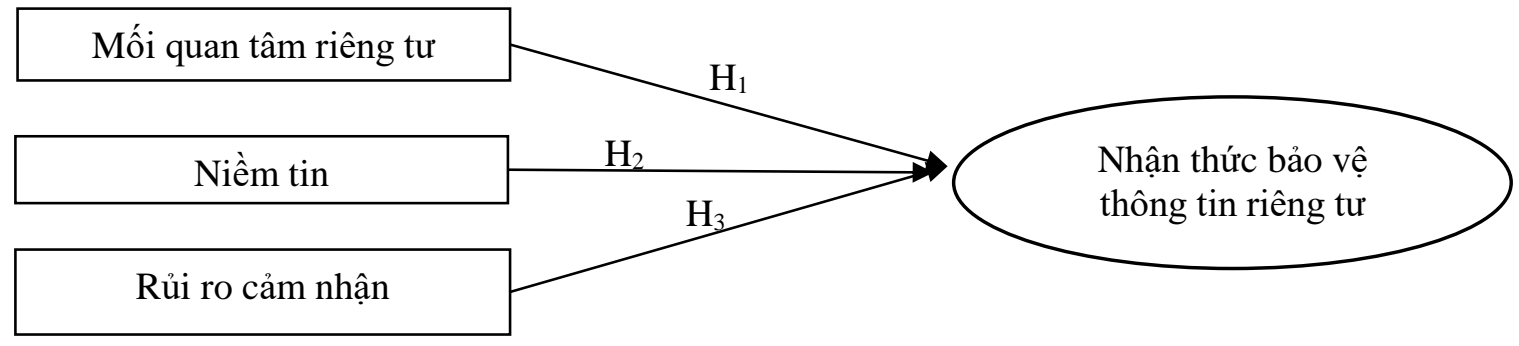

Hình 1. Mô hình nghiên cứu

\section{PHƯƠNG PHÁP NGHIÊN CÚU}

\subsection{Thu thập dữ liệu và mẫu nghiên cứu}

Tác giả đã tiến hành thảo luận nhóm tại hai khu vực Trường Đại học Công nghiệp TP.HCM và quận 1. Tại Trường Đại học Công nghiệp TP.HCM, cuộc thảo luận diễn ra với sự tham gia đóng góp của năm giảng viên giảng dạy về môn thương mại điện tử của khoa Quản trị kinh doanh cũng như an toàn thông tin của khoa Công nghệ thông tin, đây là những cá nhân am hiểu về thương mại điện tử cũng như lĩnh vực bảo vệ thông tin riêng tư. Thảo luận nhóm tại quận 1 với sự tham gia của năm khách hàng thường xuyên mua hàng trực tuyến gồm nhân viên văn phòng, sinh viên và nhân viên công nghệ thông tin. Qua thảo luận nhóm ở hai địa điểm, tác giả rút ra được kết luận như sau: khách hàng có nhận thức bảo vệ thông tin bởi vì sự ảnh hưởng của các yếu tố sau (1) sự quan tâm riêng tư của chính bản thân họ, (2) niềm tin của họ đối với nhà cung cấp dịch vụ hay trang web, (3) rủi ro cảm nhận, (4) tính giải trí của việc mua hàng trực tuyến và (5) tính dễ dùng của các trang web bán hàng. Trong đó các yếu tố từ (1) đến (3) được nhiều người lựa chọn sẽ được sử dụng để nghiên cứu. Sau khi nghiên cứu định tính, tác giả đã thiết kế bản câu 
hỏi dựa trên kết quả định tính và các nghiên cứu trước. Tiếp đó đã khảo sát sơ bộ với 30 mẫu, phân tích nhân tố khám phá (EFA) và kiểm định độ tin cậy của thang đo sơ bộ cho kết quả là các thang đo hợp lệ để khảo sát chính thức.

Vì đây là mô hình nghiên cứu khá mới nên để đảm bảo tính thiết thực của nghiên cứu tác, giả tiến hành thiết kế bản câu hỏi liên quan tới nội dung khảo sát trực tiếp và thu nhận lại các phiếu đã phát đi nhằm thu thập những ý kiến, đánh giá và phản ánh chân thực nhận thức bảo về thông tin riêng tư của khách hàng khi mua hàng trực tuyến tại Tp. Hồ Chí Minh. Tuy nhiên, kích thước mẫu sẽ cho kết quả nghiên cứu tương ứng: 50 là rất kém, 100 kém, 200 là khá tốt, 300 là tốt, 500 là rất tốt và 1000 là tuyệt vời [29]. Bài nghiên cứu sử dụng cỡ mẫu trong khảo sát chính thức là 200 người. Số lượng phiếu khảo sát thu lại là 198 mẫu (tỷ lệ hồi đáp 99\%), trong đó: 192 mẫu hợp lệ và 6 mẫu không hợp lệ (lý do do đáp viên trả lời không đầy đủ thông tin). Trong tổng số 198 mẫu khảo sát thu lại, số người trả lời có tham gia mua hàng trực tuyến là 192 đáp viên, chiếm $97 \%$. Số người trả lời chưa mua hàng trực tuyến là 6 , chiếm $3 \%$. Đối tượng khảo sát không có sự chênh lệch quá lớn giữa tỉ lệ nam và nữ (58,9\% là nữ). Theo thống kê về nghề nghiệp số người tham gia trả lời khảo sát có $41,1 \%$ đối tuợng là học sinh-sinh viên, nghề nghiệp là nhân viên văn phòng chiếm $44 \%$, kinh doanh tự do chiếm $13,5 \%$. Giáo viên-giảng viên chiếm $9,9 \%$ chiếm tỷ lệ thấp, chiếm $12,5 \%$ là khách hàng có nghề nghiệp khác. Theo kết quả thống kê, đáp viên có thu nhập dưới 5 triệu đồng chiếm tỷ lệ cao nhất $52,1 \%$. Đáp viên thu nhập từ $5-10$ triệu đồng chiếm tỷ lệ khá cao với $34,4 \%$ chứng tỏ những người có mức thu nhập này cũng có nhu cầu mua sắm trực tuyến cao. Đáp viên có thu nhập từ 10-15 triệu đồng chiếm 9,4\%, đây cũng là những đáp viên có thu nhập cao và cũng có thể là khách hàng tiềm năng trong tương lai. Đáp viên có thu nhập trên 15 triệu đồng chiếm 4,2\% chiếm tỷ lệ thấp.

\subsection{Thang đo}

Thang đo của tất cả các khái niệm nghiên cứu trong bài được dựa trên các nghiên cứu trước đây, được điều chỉnh qua nghiên cứu sơ bộ và thể hiện dưới dạng các phát biểu. Các thang đo sử dụng thang đo Likert với 5 mức độ từ (1) Hoàn toàn không đồng ý đến (5) Hoàn toàn đồng ý.

Bảng 1. Thống kê các nhân tố sử dụng trong nghiên cứu

\begin{tabular}{|l|c|l|}
\hline Nhóm nhân tố & Số lượng biến & Nghiên cứu sử dụng \\
\hline Quan tâm về riêng tư & 4 & $\begin{array}{l}\text { Heng Xu và công sư (2008) } \\
\text { Nghiên cứu định tính để điều chỉnh }\end{array}$ \\
\hline Niểm tin & 4 & $\begin{array}{l}\text { Anil Gurung, Xin Luo, M.K Raja (2008) } \\
\text { Nghiên cứu định tính để điều chỉnh }\end{array}$ \\
\hline Rủi ro cảm nhận & 4 & $\begin{array}{l}\text { Heng Xu và công sư (2008) } \\
\text { Anil Gurung, Xin Luo, M.K Raja (2008) } \\
\text { Nghiên cứu định tính để điều chỉnh }\end{array}$ \\
\hline Nhận thức bảo vệ thông tin riêng tư & 4 & $\begin{array}{l}\text { Anil Gurung, Xin Luo, M.K Raja (2008) } \\
\text { Hu và Dinev (2005) } \\
\text { Nghiên cứu định tính để điều chỉnh }\end{array}$ \\
\hline
\end{tabular}

Nguồn: Thống kê của tác giả

\section{KÊTT QUẢ NGHIÊN CÚ́U}

\subsection{Thống kế mô tả}

Đánh giá về mức độ quan trọng của việc bảo vệ thông tin ở mức quan trọng là $37 \%$ chiếm tỷ lệ cao, mức rất quan trọng chiếm 20,3\%. Tầm quan trọng ở mức bình thường chiếm $33,9 \%$ cũng chiếm tỷ lệ cao, mức độ rất không quan trọng $(0,5 \%)$ và không quan trọng $(8,3 \%)$ chiếm tỷ lệ thấp. Từ đó, chúng ta có thể thấy nhận thức về việc bảo thông tin của khách hàng là tương đối cao (trên $50 \%$ ).

Theo như kết quả thống kê chỉ ra rằng lý do mua hàng trực tuyến của khách hàng gồm tiết kiệm thời gian chiếm $70,3 \%$, có thể lựa chọn sản phẩm mọi lúc mọi nơi chiếm $55,2 \%$, không cần trực tiếp đến cửa hàng chiếm $43,8 \%$, hàng hóa đa dạng chiếm $34,4 \%$. Có thể nói, lý do mua hàng trực tuyến chủ yếu là sự tiện lợi và linh hoạt về thời gian khi mua hàng trực tuyến. 
Những thông tin mà đáp viên lo lắng phải cung cấp thông tin khi mua sắm trực tuyến chiếm tỷ lệ cao nhất là mất hay lộ số thẻ tín dụng, thẻ ghi nợ, $\mathrm{ATM}$ chiếm $65,6 \%$ và thông tin về số điện thoại chiếm $59,4 \%$. Những thông tin chiếm tỷ lệ cao vì đây là những thông tin quan trọng cần được bảo mật đối với khách hàng khi cung cấp thông tin trong giao dịch trực tuyến. Bên cạnh đó, các thông tin mà khách hàng lo lắng khi cung cấp như thu nhập chiếm $37 \%$, địa chỉ email $17,7 \%$ cũng chiếm tỷ lệ cao. Trình độ học vấn $(10,4 \%)$ và tuổi tác $(12 \%)$ những thông tin này chiếm tỷ lệ thấp hơn nhưng cũng là một trong những lo lắng của khách hàng khi phải cung cấp thông tin của họ.

Bảng 2. Kết quả thống kê mô tả

\begin{tabular}{|c|c|c|}
\hline \multirow{5}{*}{$\begin{array}{c}\text { Tầm quan trọng } \\
\text { của bảo vệ thông } \\
\text { tin khi mua hàng } \\
\text { trực tuyến }\end{array}$} & Rất không quan trọng & $0,5 \%$ \\
\hline & Không quan trọng & $8,3 \%$ \\
\hline & Bình thường & $33,9 \%$ \\
\hline & Quan trọng & $37 \%$ \\
\hline & Rất quan trọng & $20,3 \%$ \\
\hline \multirow{5}{*}{$\begin{array}{l}\text { Lý do mua hàng } \\
\text { trụ̣c tuyến }\end{array}$} & Tiết kiệm thời gian & $\mathbf{7 0 , 3 \%}$ \\
\hline & Hàng hóa đa dạng. & $34,4 \%$ \\
\hline & Tiết kiệm được chi phí. & $19,8 \%$ \\
\hline & $\begin{array}{l}\text { Không cần trực tiếp đến } \\
\text { cửa hàng. }\end{array}$ & $43,8 \%$ \\
\hline & $\begin{array}{l}\text { Có thể lựa chọn sản phẩm } \\
\text { mọi lúc mọi nơi. }\end{array}$ & $55,2 \%$ \\
\hline
\end{tabular}

\begin{tabular}{|c|c|c|}
\hline \multirow{6}{*}{$\begin{array}{c}\text { Nhũ̃ng } \\
\text { thông tin } \\
\text { riêng tư } \\
\text { khách hàng } \\
\text { lo lắng khi } \\
\text { cung cấp để } \\
\text { mua hàng } \\
\text { trực tuyến }\end{array}$} & Thu nhập. & $37 \%$ \\
\hline & Địa chỉ email. & $17,7 \%$ \\
\hline & Số điện thoại. & $\mathbf{5 9 , 4 \%}$ \\
\hline & Tuổi tác. & $12 \%$ \\
\hline & Trình độ học vấn. & $10,4 \%$ \\
\hline & $\begin{array}{l}\text { Số thẻ tín dụng, thẻ } \\
\text { ghi nợ, ATM. }\end{array}$ & $65,6 \%$ \\
\hline
\end{tabular}

Nguồn: Kết quả xủ lý SPSS của tác giả

Về nhận thức bảo vệ thông tin của khách hàng, việc khách hàng hiểu được các vấn đề bảo vệ thông tin riêng tư trong xã hội hiện nay được đồng ý thấp nhất 2,43 và cao nhất là khách hàng luôn sử dụng các phần mềm chống virus để bảo vệ máy tính với sự đồng ý trung bình là 2,71 . Nhìn chung, mức đồng ý về các yếu tố thể hiện sự nhận thức bảo vệ thông tin rất thấp (trung bình của tất cả các yếu tố đều dưới mức 3 ).

Bảng 3. Bảng giá trị trung bình của nhân tố về nhận thức bảo vệ thông tin riêng tư

\begin{tabular}{|c|c|c|}
\hline Thang đo & Mã hóa & Trung bình \\
\hline \multirow{3}{*}{ Nhận thức bảo vệ thông tin riêng tư } & PPIP1 & 2,71 \\
\cline { 2 - 3 } & PPIP2 & 2,60 \\
\cline { 2 - 3 } & PPIP3 & 2,56 \\
\cline { 2 - 3 }
\end{tabular}

Nguồn: Kết quả xủ lý SPSS của tác giả

\section{2. Đánh giá độ tin cậy của thang đo và phân tích nhân tố}

Tiếp theo, tác giả tiến hành phân tích độ tin cậy của thang đo thông qua công cụ là hệ số Cronbach's Alpha. Hệ số Cronbach's Alpha được sử dụng nhằm loại bỏ các biến không phù hợp. Cronbach's Alpha của thang đo $>0,95$ là các biến quan sát không có sự khác biệt nhau, Cronbach's Alpha $>0,6$ là thang đo tin cậy và $0,7<$ Cronbach's Alpha $<0,8$ là thang đo lý tưởng. Các biến có hệ số tương quan biến tổng nhỏ hơn 0,3 sẽ bị loại $[14,31]$.

Các nhóm nhân tố đều đạt yêu cầu vì hệ số tương quan biến tổng > 0,3 , hệ số Cronbach's Alpha > 0,7. Riêng nhóm thang đo "Niềm tin" có 1 biến TR2 (Công ty trực tuyến có quyền truy cập vào các thông tin riêng tư để xử lý các giao dịch một cách hợp lý) có hệ số tương quan biến tổng $0.245<0.3$ và hệ số Cronbach's Alpha nếu loại biến bằng 0,704 > hệ số Cronbach's Alpha là 0,637 nên ta loại biến TR2 và tiến hành chạy lại. Sau khi chạy lại ta được kết quả hệ số Cronbach's Alpha > 0,7, hệ số tương quan biến tổng > 0,3 và hệ số Cronbach's Alpha nếu loại biến nhỏ hơn hệ số Cronbach's Alpha nên thang đo đạt tiêu chuẩn và có ý nghĩa thống kê. Tương tự, nhóm nhận thức bảo vệ thông tin riêng tư có biến PIP4 (Anh/ chị hiểu được các vấn đề bảo vệ thông tin riêng tư trong xã hội hiện nay) có hệ số Cronbach's Alpha nếu xóa biến là 0,701 lớn hơn hệ số Cronbach's Alpha của cả nhóm là 0,683 nên ta xóa biến này. 
Bảng 4. Kiểm định mức độ tin cậy của thanh đo

\begin{tabular}{|c|c|c|c|c|c|c|c|}
\hline $\begin{array}{l}\text { NHẤN } \\
\text { TỐ }\end{array}$ & $\begin{array}{c}\text { M̃̃ } \\
\text { HÓA }\end{array}$ & $\begin{array}{c}\text { Hệ số } \\
\text { tương } \\
\text { quan biến } \\
\text { tổng }\end{array}$ & $\begin{array}{c}\text { Hệ số } \\
\text { Cronbach's } \\
\text { Alpha nếu loại } \\
\text { biến } \\
\end{array}$ & NHÂN TỐ & $\begin{array}{l}\text { M̃̃ } \\
\text { HÓA }\end{array}$ & $\begin{array}{c}\text { Hệ số } \\
\text { tương } \\
\text { quan biến } \\
\text { tổng } \\
\end{array}$ & $\begin{array}{c}\text { Hệ số } \\
\text { Cronbach's } \\
\text { Alpha nếu loại } \\
\text { biến }\end{array}$ \\
\hline \multirow{5}{*}{$\begin{array}{c}\text { MỐI } \\
\text { QUAN } \\
\text { TÂM } \\
\text { RIÊNG } \\
\text { TU' (PC) }\end{array}$} & \multicolumn{3}{|c|}{ Hệ số Cronbach's Alpha $(\mathrm{PC})=\mathbf{0 , 8 0 6}$} & \multirow{5}{*}{$\begin{array}{c}\text { RỦI RO } \\
\text { CẢM } \\
\text { NHẦN } \\
\text { (PR) }\end{array}$} & \multicolumn{3}{|c|}{ Hệ số Cronbach's Alpha $(P R)=0,814$} \\
\hline & $\mathrm{PC} 1$ & 0,532 & 0,797 & & PR1 & 0,614 & 0,775 \\
\hline & $\mathrm{PC} 2$ & 0,619 & 0,758 & & PR2 & 0,740 & 0,713 \\
\hline & PC3 & 0,679 & 0,728 & & PR3 & 0,635 & 0,767 \\
\hline & $\mathrm{PC} 4$ & 0,662 & 0,738 & & PR4 & 0,552 & 0,802 \\
\hline \multirow{4}{*}{$\begin{array}{l}\text { NIỀM } \\
\text { TIN } \\
(\text { TR) }\end{array}$} & \multicolumn{3}{|c|}{ Hệ số Cronbach's Alpha $(\mathrm{TR})=0,704$} & $\begin{array}{l}\text { NHẤN } \\
\text { THÚC }\end{array}$ & \multicolumn{3}{|c|}{$\begin{array}{c}\text { Hệ số Cronbach's Alpha (PPIP) = } \\
0,701\end{array}$} \\
\hline & TR1 & 0,448 & 0,698 & BẢO VÊ & PPIP1 & 0,543 & 0,578 \\
\hline & TR3 & 0,595 & 0,532 & $\begin{array}{c}\text { THONG } \\
\text { TIN }\end{array}$ & PPIP2 & 0,553 & 0,569 \\
\hline & TR4 & 0,545 & 0,596 & $\begin{array}{c}\text { RIENGG } \\
\text { TU' (PPIP) }\end{array}$ & PPIP3 & 0,465 & 0,685 \\
\hline
\end{tabular}

Nguồn: Kết quả xủ lý SPSS của tác giả

Qua kết quả kiểm định phân tích nhân tố, ta thấy hệ số KMO có giá trị $=0,805>0,5$. Giá trị sig. Barlett's Test $=0,00<0,05$ do đó phân tích nhân tố hợp lý và hệ số phân tích đạt yêu cầu. Các biến có tương quan với nhau trong tổng thể. Eigenvalues $=1,212>1$ đại diện cho phần biến thiên được giải thích bởi mỗi nhân tố và nhân tố rút ra có ý nghĩa tóm tắt thông tin tốt nhất. Tổng phương sai trích $=65,211 \%$ $>50 \%$. Điều này chứng tỏ $65,211 \%$ biến thiên của dữ liệu được giải thích bởi ba nhân tố.

Bảng 5. Phân tích nhân tố EFA

\begin{tabular}{|c|c|c|c|c|c|c|c|}
\hline \multirow{2}{*}{ Biến độc lập } & \multicolumn{4}{|c|}{ Nhân tố } & \multicolumn{3}{|c|}{$K M O=0,805$ (sig. $=0,00)$} \\
\hline & 1 & 2 & 3 & \begin{tabular}{|l|l|}
4 & 5 \\
\end{tabular} & & & \\
\hline & & & & & Eigenvalues & $\begin{array}{c}\text { Phương sai trích } \\
(\%)\end{array}$ & Nhân tố \\
\hline $\begin{array}{l}\text { PC4 } \\
\text { PC2 } \\
\text { PC3 } \\
\text { PC1 }\end{array}$ & $\begin{array}{l}0,822 \\
0,795 \\
0,793 \\
0,607\end{array}$ & & & & 4,011 & 36,464 & $\begin{array}{l}\text { Quan tâm về riêng } \\
\text { tu' }\end{array}$ \\
\hline $\begin{array}{l}\text { PR4 } \\
\text { PR3 } \\
\text { PR2 } \\
\text { PR1 } \\
\end{array}$ & & $\begin{array}{l}0,840 \\
0,772 \\
0,725 \\
0,677\end{array}$ & & & 1,950 & 54,194 & Nhận thúcc rủi ro \\
\hline $\begin{array}{l}\text { TR3 } \\
\text { TR4 } \\
\text { TR1 }\end{array}$ & & & $\begin{array}{l}0,836 \\
0,813 \\
0,722\end{array}$ & & 1,212 & 65,211 & Niềm tin \\
\hline $\begin{array}{l}\text { Biến phụ } \\
\text { thuộc }\end{array}$ & \multicolumn{7}{|c|}{$\begin{array}{l}\text { Hệ số KMO =0,709 } \\
\text { Giá trị Sig trong kiểm định Barlett }=0,000\end{array}$} \\
\hline $\begin{array}{l}\text { PPIP2 } \\
\text { PPIP1 } \\
\text { PPIP3 }\end{array}$ & $\begin{array}{l}0,820 \\
0,815 \\
0,744\end{array}$ & & & & 2,103 & 52,585 & $\begin{array}{l}\text { Nhận thức bảo vệ } \\
\text { thông tin riêng tur }\end{array}$ \\
\hline
\end{tabular}

Nguồn: Kết quả xủ lý SPSS của tác giả

Đối với biến phụ thuộc: Kiểm định KMO có giá trị 0,709 thỏa điều kiện $0,5 \leq \mathrm{KMO} \leq 1$. Do vậy, phân tích nhân tố phù hợp với dữ liệu thực tế. Kiểm định Bartlett (Bartlet's Test) có giá trị sig $=0,00<$ 0,05 . Do vậy, các biến quan sát có tương quan với nhau trong mỗi nhóm nhân tố. Tổng phương sai trích $=$ $52,584 \%>50 \%$. Điều này chứng tỏ 52,584\% biến thiên của dữ liệu được giải thích bởi một nhân tố. 


\subsection{Phân tích tương quan và phương trình hồi quy}

Xem xét các mối quan hệ tương quan giữa biến phụ thuộc với từng biến độc lập thông qua phân tích tương quan Pearson. Nểu hệ số tương quan giữa biến phụ thuộc và biến độc lập lớn chứng tỏ giữa chúng có mối quan hệ với nhau, và phân tích hồi quy là phù hợp. Ngược lại, nếu các biến độc lập cũng có hệ số tương quan với nhau lớn thì có thể xảy ra hiện tượng đa cộng tuyến trong mô hình hồi quy đang xem xét. Theo bảng 6 , tất cả các hệ số tương quan giữa biến phụ thuộc và các biến độc lập đều từ 0,419 đến 0,520 nên nhận thức bảo vệ thông tin riêng tư (PPIP) có sự tương quan với các biến độc lập với độ tin cậy $99 \%$

Ta có $\mathrm{R}^{2}=0,472$, điều này cho thấy sự phù hợp của mô hình với biến quan sát khá tốt và biến "Nhận thức bảo vệ thông tin thông tin" được giải thích bởi ba nhân tố ở trên và $47,2 \%$ sự biến thiên của PPIP được giải thích ba biến độc lập. Theo nhiều lý thuyết, $\mathrm{R}^{2}$ phải lớn hơn $50 \%$ nhưng nếu mô hình dùng để dự báo thì mới cần điều kiện trên còn nếu với mục đích đánh giá mức độ ảnh hưởng thì $\mathrm{R}^{2}$ không có nhiều ý nghĩa [9].

Hệ số Durbin Watson dùng để kiểm định tương quan chuỗi bậc nhất cho thấy mô hình không vi phạm khi sử dụng phương pháp hồi quy bội vì giá trị Durbin Watson đạt được là 2,015 (nằm trong khoảng từ 0 đến 4 ) và chấp nhận giả thuyết không có sự tương quan chuỗi bậc nhất trong mô hình. Như vậy, mô hình hồi quy bội thỏa các điều kiện đánh giá và kiểm định độ phù hợp cho việc rút ra các kết quả nghiên cứu.

Kiểm định $\mathrm{F}$ sử dụng trong phân tích phương sai là một phép kiểm định giả thuyết về độ phù hợp của mô hình hồi quy tuyến tính tổng thể để xem xét biến phụ thuộc có liên hệ tuyến tính với toàn bộ tập hợp của các biến độc lập. Qua bảng phân tích phương sai ANOVA cho thấy trị số $\mathrm{F}=56,701$ và có mức ý nghĩa sig. $=0,000$ (sig. $\leq 0,05)$, có ý nghĩa mô hình hồi quy phù hợp với dữ liệu thu thập được và các biến đưa vào đều có ý nghĩa trong thống kê với mức ý nghĩa $5 \%$.

Hệ số phóng đại phương sai VIF $<10$ cho thây không có hiện tượng đa cộng tuyến do các biến độc lập không có mối quan hệ chặt chẽ với nhau. Các giá trị Sig. $<0,05$ nên hệ số hồi quy đều có ý nghĩa về mặt thống kê.

Bảng 6. Kết quả hồi quy tuyến tính

\begin{tabular}{|c|c|c|c|c|c|c|c|c|c|}
\hline \multirow{2}{*}{ Mô hình } & \multicolumn{2}{|c|}{$\begin{array}{l}\text { Hệ số không } \\
\text { chuẩn hóa }\end{array}$} & \multirow{2}{*}{$\begin{array}{c}\text { Hệ số chuẩn } \\
\text { hóa }\end{array}$} & \multirow{2}{*}{$\mathrm{t}$} & \multirow{2}{*}{ Sig. } & \multicolumn{2}{|c|}{ Đa cộng tuyến } & \multirow[t]{2}{*}{$\begin{array}{c}\text { Hệ số } \\
\text { tương quan } \\
\text { với biến Y }\end{array}$} & \multirow[t]{2}{*}{$\begin{array}{c}\text { Kiểm định giả } \\
\text { thuyết }\end{array}$} \\
\hline & B & $\begin{array}{l}\text { Std. } \\
\text { Error }\end{array}$ & & & & Tolerance & VIF & & \\
\hline (Constant) & 2.765 & 0,175 & & 15.762 & 0,000 & & & & \\
\hline $\mathrm{PC}$ & 0,120 & 0,043 & 0,176 & 2.767 & 0,006 & 0,693 & 1.443 & 0,419 & Chấp nhận $\mathrm{H}_{1}$ \\
\hline TR & $-0,342$ & 0,039 & $-0,464$ & -8.700 & 0,000 & 0,988 & 1.012 & $-0,502$ & Chấp nhận $\mathrm{H}_{2}$ \\
\hline PR & 0,243 & 0,044 & 0,351 & 5.548 & 0,000 & 0,700 & 1.429 & 0,472 & Chấp nhận $\mathrm{H}_{3}$ \\
\hline \multicolumn{3}{|c|}{$\mathrm{R}^{2}$} & \multicolumn{5}{|c|}{0,472} & & \\
\hline \multicolumn{3}{|c|}{$\mathrm{F}($ sig. $=0.00)$} & \multirow{2}{*}{\multicolumn{5}{|c|}{56,701}} & & \\
\hline \multicolumn{3}{|c|}{ Durbin - Watson } & & \multicolumn{4}{|c|}{2,015} & & \\
\hline
\end{tabular}

Nguồn: Kết quả xủ lý SPSS của tác giả

Theo kết quả bảng 6 cho thấy Sig. của các hệ số hồi quy Beta < 0,05 nên chấp nhận các hệ số Beta.

Nhân tố mối quan tâm riêng tư $(\beta=0,120)$, dấu $(+)$ : Quan hệ đồng biến. Khi nhân tố mối quan tâm riêng tư tăng/giảm lên 1 đơn vị thì nhận thức bảo vệ thông tin riêng tư tăng/giảm trung bình 0,120 đơn vị.

Nhân tố niềm tin $(\beta=-0,342)$, dấu (-): Quan hệ nghịch biến. Khi nhân tố niềm tin tăng/giảm lên 1 đơn vị thì nhận thức bảo vệ thông tin riêng tư giảm/tăng trung bình 0,342 đơn vị và ngược lại.

Nhân tố rủi ro cảm nhận $(\beta=0,243)$, dẩu $(+)$ : Quan hệ đồng biến. Khi nhân tố rủi ro cảm nhận tăng/giảm 1 đơn vị thì nhận thức bảo vệ thông tin riêng tư tăng/giảm trung bình 0,243 đơn vị. 


\section{KẾT LUẬN VÀ HÀM Ý QUẢN TR!}

Thứ nhất, theo phương trình hồi quy ta có thể kết luận nhận thức bảo vệ thông tin riêng tư bị tác động bởi các yếu tố như mối quan tâm riêng tư, niềm tin và cảm nhận rủi ro. Trong đó mối quan tâm riêng tư cũng như cảm nhận rủi ro tác động đồng biến với nhận thức bảo vệ thông tin riêng tư, còn niềm tin sẽ tác động nghịch biến. Có nghĩa là khi mối quan tâm riêng tư và cảm nhận rủi ro của khách hàng càng cao thì họ thường nhận thức bảo vệ thông tin riêng tư cao, còn nếu như khách hàng tin tưởng vào nhà cung cấp dịch vụ thì họ giảm nhận thức bảo vệ thông tin riêng tư khi cung cấp thông tin và thực hiện giao dịch trực tuyến. Theo hệ số hồi quy chuẩn hóa, yếu tố niềm tin tác động nhiều nhất đến nhận thức bảo vệ thông tin riêng tư (độ lớn Beta $=0,464)$, tiếp theo là nhận thức rủi ro $(0,351)$ và cuối cùng là yếu tố quan tâm đến riêng tư $(0,176)$. Thứ hai, theo kết quả thống kê mô tả, người dùng rất quan tâm đến vấn đề bảo vệ thông tin riêng tư khi mua hàng trên mạng (trên 57,3\% trả lời là việc đó là quan trọng và rất quan trọng) nhưng họ lại không nhận thức được các biện pháp bảo vệ thông tin riêng tư cũng như nâng cao sự hiểu biết về các vấn đề riêng tư (các yếu tố nhận thức bảo vệ thông tin riêng tư đều dưới mức trung bình). Một nghịch lý về Quyền riêng tư đã được ghi nhận: "mặc dù trong các tài liệu thu thập khẳng định rằng mối quan tâm của người dùng Internet về bảo mật cao, NHU'NG người tiêu dùng vẫn dễ dàng gửi thông tin riêng tư của họ trong một số trường hợp" [3]. Bên cạnh đó, những nghiên cứu trước đây về quyền riêng tư chỉ ra rằng các cá nhân sẵn sang công khai thông tin riêng tư để trao đổi những lợi ích kinh tế hay lợi ích xã hội dựa trên "tính toán về quyền riêng tư" $[18,20,28]$. Qua đó, ta có thể nhận thấy người sử dụng Internet có thể sử dụng chiến lược khác nhau để bảo vệ sự riêng tư của mình để có thể trỏ nên tự tin trong các giao dịch thương mại điện tử. Trong nghiên cứu về "Nghịch lý về quyền riêng tư: Ý định công bố thông tin riêng tư đối lập với hành vi”, PA Norberg và cộng sự (2007) có nói rằng khách hàng lựa chọn và thưởng dễ dãi trong việc bảo vệ các thông tin riêng tư của chính họ [23].

Qua kết quả nghiên cứu đạt được, tác giả có một số gợi ý cho các doanh nghiệp trực tuyến nhằm giảm bớt sự bảo vệ thông tin của khách hàng, điều có thể ảnh hưởng ý định giao dịch của khách hàng.

Tăng niềm tin cho khách hàng bằng cách sau đây: (1) Nâng cao năng lực quản lý của lãnh đạo và kỹ năng chuyên môn các nhân viên của các công ty trực tuyến để thực hiện giao dịch với khách hàng. Cụ thể: tham gia các khóa đào tạo, các lớp bồi dưỡng về các vấn đề bảo vệ thông tin khách hàng và kinh doanh trực tuyến cho các cấp lãnh đạo và nhân viên; tổ chức các buổi nói chuyện giữa lãnh đạo và nhân viên nhằm trao đổi kinh nghiệm làm việc, tổ chức các buổi tập huấn về kỹ năng chăm sóc, tư vấn khách hàng. (2) Đảm bảo cho lợi ích của khách hàng khi cung cấp thông tin: công ty sẽ liên tục cập nhật và sửa đổi các quy định về hệ thống quản lý để xử lý và bảo vệ thông tin riêng tư, đảm bảo sự tuân thủ của các nhân viên công ty với những quy định và hệ thống đó, các công ty cần xây dựng những chính sách hỗ trợ khách hàng thật tốt để tạo nên sự uy tín, thu hút và gây thiện cảm cho khách hàng. (3) Các công ty trực tuyến phải công bằng, rõ ràng trong cách ứng xử, làm việc của mình: Website có mục thông tin doanh nghiệp rõ ràng: tên công ty, giấy phép kinh doanh, địa điểm kinh doanh, trụ sở, số điện thoại hỗ trợ, thông tin giới thiệu doanh nghiệp rõ ràng, đăng ký với Bộ Công Thương: có chứng nhận đăng ký Sàn giao dịch thương mại điện tử với Bộ Công Thương và hiển thị rõ ràng trên website bán hàng.

Bên cạnh đó, các doanh nghiệp nên có chính sách để giảm rủi ro cảm nhận của khách hàng khi mua hàng: (1) Đảm bảo sự riêng tư về thông tin riêng tư của khách hàng: doanh nghiệp cần phải thiết lập quyền riêng tư cá nhân cho khách hàng thông qua những tài khoản đăng nhập cá nhân giúp khách hàng có thể tự mình thiết lập tự động về những thông tin cần bảo mật, những thông tin cho phép hoặc không cho phép truy cập để đảm bảo cho sự riêng tư của khách hàng nhằm tránh việc bị kẻ gian lừa đảo, hoặc bị lấy cắp thông tin sử dụng cho những mục đích không chính đáng; doanh nghiệp nên cho khách hàng xem quá trình bảo mật thông tin của khách hàng nhằm đảm bảo thông tin của khách hàng không bị rò rỉ ra bên ngoài. (2) Doanh nghiệp đảm bảo việc cung cấp thông tin riêng tư sẽ không phát sinh nhiều vấn đề không mong đợi: doanh nghiệp đưa ra những quy định để đảm bảo quy trình thu thập thông tin của khách hàng là bảo mật tuyệt đối, quá trình thu thập thông tin diễn ra nhanh chóng, không tốn nhiều thời gian của khách hàng, thông tin riêng tư của khách hàng sẽ được kiểm tra kỹ càng, tránh sai sót trong quá trình cung cấp. (3) Doanh nghiệp hạn chế thấp nhất các rủi ro khi cung cấp thông tin riêng tư: doanh nghiệp cần phải có những hình thức bảo mật an toàn cho khách hàng tránh việc thông tin khách hàng bị đánh cắp, lừa đảo; kho dữ liệu về thông tin riêng tư về khách hàng của doanh nghiệp nên được cập nhật những phần mềm mới nhất, có tính bảo mật cao nhất.(4) Đảm bảo thông tin riêng tư của khách hàng được sự dụng đúng 
mục đích: những thông tin liên quan đến việc cung cấp thông tin của khách hàng cần phải đưa ra những mục đích thu thập như cung cấp dịch vụ, tiếp thị, thông tin với khách hàng về sản phẩm và dịch vụ, khảo sát nghiên cứu thị trường.

Hơn nữa, đối với thị trường Việt Nam, các công ty kinh doanh trực tuyến cũng phải biết cách để làm giảm mối quan tâm về riêng tư của khách hàng khi thực hiện giao dịch trực tuyến. (1) Doanh nghiệp cần thiết lập cách bảo vệ thông tin riêng tư của khách hàng: Có chế độ bảo vệ thông tin, xác định, thống kê được các hoạt động và giao dịch bất thường phát sinh trong hệ thống liên quan đến sử dụng thông tin của khách hàng; lập ra một bảng cam kết về các chính sách bảo mật thông tin cho khách hàng đề khách hàng yên tâm và giảm bớt lo lắng, lo ngại khi thực hiện các giao dịch trực tuyến, trao đổi thông tin. (2) Doanh nghiệp cần có chế độ bảo mật phù hợp trong giao dịch với khách hàng: có những quy định về bảo mật thông tin đối với khách hàng, có những hướng dẫn khách hàng khi cung cấp thông tin, chủ động trong việc giải quyết những vấn đề lạm dụng thông tin khách hàng. (3) Doanh nghiệp chỉ thu thập những thông tin nào cần thiết cho việc giao dịch với khách hàng như số điện thoại, địa chỉ để phục vụ cho việc giao hàng.

Hạn chế của nghiên cứu và hướng nghiên cứu tiếp theo

Mặc dù kết quả nghiên cứu đã có những đóng góp nhất định về mặt học thuật cũng như thực tiễn nhưng nghiên cứu của tác giả cũng gặp một số hạn chể nhất định: Số lượng nhân tố trong mô hình vẫn còn tương đối ít ( 3 nhân tố tác động) dẫn đến việc hệ số $\mathrm{R}^{2}$ chưa đạt được $50 \%$, nghiên cứu được thực hiện theo phương pháp chọn mẫu thuận tiện và kích thước mẫu tương đối nhỏ nên khả năng khái quát hóa không cao. Điều này dẫn đến việc nghiên cứu sẽ không phản ánh đầy đủ và chính xác các nhận thức, đánh giá về nhận thức bảo vệ thông tin khi giao dịch thương mại điện tử. Bài báo cũng chỉ mới sử dụng mô hình hồi quy để phân tích mối quan hệ giữa các biến độc lập và phụ thuộc một cách tương đối đơn giản. Hơn nữa, thời gian tác giả tìm hiểu, khảo sát, làm bài và hoàn thiện bài nghiên cứu tương đối ngắn cho nên nghiên cứu có thể có một số sai sót nhất định.

Hướng nghiên cứu tiếp theo đó là tăng thêm các nhân tố tác động đến nhận thức bảo vệ thông tin riêng tư của khách hàng khi giao dịch trực tuyến để hiểu rõ hơn nguyên nhân tác động tạo ra nhận thức trong khách hàng về vấn đề này. Mở rộng phạm vi nghiên cứu sang các ngành dịch vụ như ngân hàng điện tử, mạng xã hội. Mở rộng phạm vi khảo sát ra các tỉnh lân cận hay các thành phố lớn để đánh giá mức độ đồng bộ về nhận thức bảo vệ thông tin riêng tư. Và sử dụng mô hình cấu trúc tuyến tính để nghiên cứu rộng hơn về nhận thức bảo vệ thông tin riêng tư cũng như ý định giao dịch trực tuyến.

\section{TÀI LIỆU THAM KHẢO}

1. Bauer, R.A., Consumer behavior as risk taking. Dynamic marketing for a changing world, 1960. 398.

2. Bauer, R.A. and D.F. Cox, Risk taking and information handling in consumer behavior. Boston: Harvard University, 1967: p. 469-486.

3. Bennett, C.J., Regulating privacy: Data protection and public policy in Europe and the United States. 1992: Cornell University Press.

4. Bộ_Công_Thương, Báo cáo thuơng mại điện tủ 20152016.

5. Burns, A.C. and R.F. Bush, Marketing research. Globalization, 2000. 1(7).

6. Cooper, D., Psychology, risk and safety. Professional Safety, 2003. 48(11): p. 39-46.

7. Cooperation, A.-P.E., APEC privacy framework. Asia Pacific Economic Cooperation Secretariat, 2005.81.

8. Culnan, M.J. and P.K. Armstrong, Information privacy concerns, procedural fairness, and impersonal trust: An empirical investigation. Organization science, 1999. 10(1): p. 104-115.

9. Chatterjee, S. and A.S. Hadi, Regression analysis by example. 2015: John Wiley \& Sons.

10. Christofides, E., A. Muise, and S. Desmarais, Information disclosure and control on Facebook: are they two sides of the same coin or two different processes? CyberPsychology \& Behavior, 2009. 12(3): p. 341-345.

11. Dinev, T. and Q. Hu, The centrality of awareness in the formation of user behavioral intention toward protective information technologies. Journal of the Association for Information Systems, 2007. 8(7): p. 386.

12. Fibikova, J.V.-L., IT Users 'Perception of Information Security. 
13. Gurung, A., X. Luo, and M. Raja, An empirical investigation on customer's privacy perceptions, trust and security awareness in E-commerce environment. Journal of Information Privacy and Security, 2008. 4(1): p. $42-60$.

14. Hair, J.F., Multivariate data analysis. 2009.

15. Howard, P.N. and S. Jones, Society online: The Internet in context. 2004: Sage.

16. $\mathrm{Hu}, \mathrm{Q}$. and T. Dinev, Is spyware an internet nuisance or public menace? Communications of the ACM, 2005. 48(8): p. 61-66.

17. Jarvenpaa, S.L., N. Tractinsky, and L. Saarinen, Consumer trust in an internet store: a cross-cultural validation. Journal of Computer-Mediated Communication, 1999. 5(2): p. 0-0.

18. Laufer, R.S. and M. Wolfe, Privacy as a concept and a social issue: A multidimensional developmental theory. Journal of social Issues, 1977. 33(3): p. 22-42.

19. Malhotra, N.K., S.S. Kim, and J. Agarwal, Internet users' information privacy concerns (IUIPC): The construct, the scale, and a causal model. Information systems research, 2004. 15(4): p. 336-355.

20. Milne, G.R. and M.E. Gordon, Direct mail privacy-efficiency trade-offs within an implied social contract framework. Journal of Public Policy \& Marketing, 1993: p. 206-215.

21. Miniwatts_Marketing_Group. 2016 08/2016]; Available from: http://www.internetworldstats.com/stats.htm.

22. Miyazaki, A.D. and A. Fernandez, Consumer perceptions of privacy and security risks for online shopping. Journal of Consumer affairs, 2001. 35(1): p. 27-44.

23. Norberg, P.A., D.R. Horne, and D.A. Horne, The privacy paradox: Personal information disclosure intentions versus behaviors. Journal of Consumer Affairs, 2007. 41(1): p. 100-126.

24. O'Malley, L., M. Patterson, and M. Evans, Intimacy or intrusion? The privacy dilemma for relationship marketing in consumer markets. Journal of Marketing Management, 1997. 13(6): p. 541-559.

25. Proctor, R.W. and J.D. Proctor, Sensation and perception. 2007: Wiley Online Library.

26. Rosen, A., The e-commerce question and answer book: a survival guide for business managers. 2002: AMACOM Div American Mgmt Assn.

27. Sitkin, S.B. and A.L. Pablo, Reconceptualizing the determinants of risk behavior. Academy of management review, 1992. 17(1): p. 9-38.

28. Stone, E.F. and D.L. Stone, Privacy in organizations: Theoretical issues, research findings, and protection mechanisms. Research in personnel and human resources management, 1990. 8(3): p. 349-411.

29. Tabachnick, B.G., L.S. Fidell, and S.J. Osterlind, Using multivariate statistics. 2001.

30. Turban, E., et al., Electronic commerce: A managerial perspective 2002. Prentice Hall: ISBN 0, 2012. 13(975285): p. 4.

31. Trọng, H. and C.N.M. Ngọc, Phân tích dĩ liệu nghiên cứu với SPSS. Nhà xuất bản Thống kê, 2005.

32. $\mathrm{Xu}, \mathrm{H}$., et al., Information privacy concerns: Linking individual perceptions with institutional privacy assurances. Journal of the Association for Information Systems, 2011. 12(12): p. 798.

33. Yenisey, M.M., A.A. Ozok, and G. Salvendy, Perceived security determinants in e-commerce among Turkish university students. Behaviour \& Information Technology, 2005. 24(4): p. 259-274.

34. Youn, S., Determinants of online privacy concern and its influence on privacy protection behaviors among young adolescents. Journal of Consumer Affairs, 2009. 43(3): p. 389-418.

Ngày nhận bài: 08/01/2017

Ngày chấp nhận đăng: 06/07/2017 